\title{
Luminal Volume Reconstruction from Angioscopic Video Images of Casts from Human Coronary Arteries
}

\author{
Johan C. H. Schuurbiers, Cornelis J. Slager, MSc, and Patrick W. Serruys, MD
}

\begin{abstract}
Intravascular angioscopy has been hampered by its limitation in quantifying obtained images. To circumvent this problem, a lightwire was used, which projects a ring of light onto the endoluminal wall in front of the angioscope. This investigation was designed to quantify luminal dimensions of casts from stenotic human coronary arteries and to investigate the feasibility of performing volume reconstruction. Angioscopic video recordings were made during simultaneous motorized pullback ( $2 \mathrm{~mm} / \mathrm{s}$ ) of an angioscopic catheter and light-emitting fiber through 4 epoxy casts from human coronary arteries. Quantification of the images was performed using a computerized cross-sectional area measurement system. The coronary casts were divided into cross-sectional segments of 4 to $10 \mathrm{~mm}$ in thickness, with the true volume of each segment determined by means of a microdispenser. Because of vessel curvature and luminal narrowing, complete visualization of the ring of light at all 1-mm-distant locations was only possible in 19 of 40 segments. For these 19 segments, linear regression analysis showed a good correlation between measured and true segmental volume $\left(r=0.97, y=0.88 x+6.58 \mathrm{~mm}^{3}\right.$, standard error of estimate $=3.48 \mathrm{~mm}^{3}$ ). The relative error in the measured segmental volumes was $3.9 \pm$ $7.1 \%$ (mean \pm SD). These initial results of endoluminal volume reconstruction demonstrate the feasibility of this technique in vitro with high accuracy and low variability, but further technical improvements are necessary to increase the success rate, especially in the quantitative assessment of vessels with complex morphology.
\end{abstract} (Am J Cardiol 1994;74:764-768)

From the Thoraxcenter, University Hospital Rolterdann-Dijkzigl, Rutterdam, The Netherlands. Manuscript received January 7, 1994; revised manuscript received and accepted May 23, 1994.

Address for reprints: Johan C.H. Schuurbiers, Laboratory for Haemodynamics, Thoraxcenter Ee 2322, University Hospital RotterdamDijkzigt, P.O. Box 1738, 3000 DR Rotterdam, The Netherlands.
$\Lambda$ ngioscopy permits the intraluminal, 3-dimensional visualization of coronary arteries and provides unique color information of the endoluminal surface that is not available from angiography or intravascular ultrasound. ${ }^{1-3}$ Consequently, angioscopy can reveal the presence of an obstructing thrombus or plaque rupture, local inflammation, minor intimal flaps, and overall endoluminal surface conditions. Therefore, angioscopy seems to be well suited for the investigation of coronary pathogenesis and endoluminal morphology, as well as the evaluation of the result of coronary interventions. ${ }^{2,4-6}$ A major drawback of angioscopy, howev$\mathrm{er}$, is its limited ability to quantify the observed images. The optical magnification of the angioscopic view depends on the distance from lens to object. Because the true positions of endoluminal surface sites are unknown, luminal dimensions cannot be determined. A method for measuring luminal diameters from angioscopic images using a ring of laser light was recently introduced to overcome this limitation. ${ }^{7}$ To extend the applicability of this technique, we investigated the feasibility of luminal area and volume reconstruction in coronary casts using a motorized pullback of the angioscope and fiber.

\section{METHODS}

Coronary casts: Five epoxy blocks with negative casts of diffusely diseased coronary arteries were obtained from a previous study. ${ }^{8}$ One cast was excluded because its small luminal diameter did not allow passage of the angioscope. During the casting procedure, the 4 coronary casts had been divided into $7,7,14$, and 12 cross-sectional segments, respectively, of 4 to $10 \mathrm{~mm}$ in thickness. From each segment, the luminal volume was obtained using a microdispenser (Microlab $\mathrm{P}$, Hamilton Bonaduz A.G., Switzerland). With the applied syringe of $1,000 \mu \mathrm{l}$, the accuracy and precision of the dispenser were $<1 \%$ and $<0.2 \%$, respectively. Segmental volumes were determined by injecting calibrated amounts of colored water and viewing the rise of the water level through the semi-transparent coronary casts to coincide with a segment boundary for each volume segment. To reduce potential misreadings originating from the meniscus on the water surface, a detergent was added to lower the surface tension. The intra- and interobserver variabilities in volume determination with the microdispenser, investigated in a previous study, ${ }^{9}$ were $0.86 \pm 1.07 \mathrm{~mm}^{3}(1.95 \pm 2.14 \%)$ and $1.0 \pm 1.41 \mathrm{~mm}^{3}(2.4$ $\pm 2.8 \%$ ), respectively, per segment.

Prior to image acquisition, endoluminal surface reflectance of the semi-transparent coronary casts was improved by adding a thin coating of white paint with a thickness $<0.02 \mathrm{~mm}$. The resulting decrease in segmental volume by the paint was estimated to be $<3.3 \%$ to 
$<2.2 \%$ for the casts with the largest and smallest mean segmental areas, respectively.

Preclslon-machlned phantoms: For the initial evaluation of the method, 2 ceramic phantoms were created, 1 with a cylindrical lumen and 1 with a cone-shaped lumen. The cylindrical phantom had 3 segments with 1$\mathrm{mm}$ increments in luminal diameter $(2,3$, and $4 \mathrm{~mm})$ each with a length of $5 \mathrm{~mm}$. The resulting segmental volumes were $15.7,35.3$, and $62.8 \mathrm{~mm}^{3}$. The coneshaped phantom had 3 segments, a central cylindrical lumen with a diameter of $2 \mathrm{~mm}$ and a length of $6 \mathrm{~mm}$, and on both sides, a truncated cone-shaped lumen with a diameter tapering from 2 to $4 \mathrm{~mm}$ and lengths of 10 and $9 \mathrm{~mm}$, respectively. This resulted in segmental volumes of 73.3, 15.7, and $65.9 \mathrm{~mm}^{3}$. Diameters of the precision-drilled holes ranged from 1 to $5 \mathrm{~mm}$ in $0.5-\mathrm{mm}$ increments.

Fiber and angioscope: Light from a red HeNe laser $(\lambda=634 \mathrm{~nm}, 8 \mathrm{~mW})$ was transmitted through a $0.25-\mathrm{mm}$ graded-index fiber containing a $0.15-\mathrm{mm}$ core and projected onto a conical mirror assembly attached to the distal end of the fiber to produce a transverse ring of laser light (Figure 1). To compensate for fiber-beam divergence, special focusing properties were added to the mirror, resulting in a $0.2-\mathrm{mm}$-wide ring at a $2.5-\mathrm{mm}$ distance from the mirror. The fiber was mounted on the angioscopic catheter (Coronary Imagecath COV45, Baxter, Irvine, California) with the ring of light positioned at $7.65 \mathrm{~mm}$ in front of the angioscope. The $4.5 \mathrm{~F}$ angioscopic catheter used in this study was composed of 3,000 imaging fibers, arranged in a circular area (diameter = $200 \mu \mathrm{m}$ ) surrounded by 10 concentrically arranged illuminating fibers (diameter $=100 \mu \mathrm{m}$ ). The viewing angle was approximately $45^{\circ}$ in water. To improve the accessibility of severe stenosis in the coronary casts, the monorail guidance port was removed from the tip of the angioscope and the fiber was directly attached to the angioscope. This resulted in a maximal tip size of 1.1 $\mathrm{mm}(3.3 \mathrm{~F})$ for angioscope and fiber. The angioscopic catheter was connected to a charge coupled device (CCD) camera system (OPTX 3000-P, Baxter) with a vertical resolution $\geq 330$ lines, and to a $300 \mathrm{~W}$ xenon light source (OPTX 300-I, Baxter).

Image acquisition and analysis: All images were acquired in water and recorded on a super VIIS recorder (Panasonic AG-7330, Panasonic, Osaka, Japan). In the coronary casts, motorized pullback $(2 \mathrm{~mm} / \mathrm{s})$ of the angioscope and fiber was started at a segment boundary, which provided a readily identifiable reference position for the ring of light to be observed from the outside of the cast. In the ceramic phantoms, the motorized pullback $(2 \mathrm{~mm} / \mathrm{s})$ was started with the ring of light just before the backplane outside of the phantoms. Identification of the video images was obtained by superimposing a digital-counter reading onto the video image indicating the elapsed time from the start of pullback.

Because of small local bends in the vessel and luminal stenoses, the ring of light was not always completely observed with the angioscope. Only those frames showing a complete visualization of the ring of light were selected for analysis. Image analysis was performed by manually delineating the ring of light using a computerized cross-sectional area measurement system. ${ }^{10}$ Figure 2 shows an example of the observed ring of light and the traced outline. Because the angioscope-mirror distance did not change during the measurements, calibration was performed only once using the $2.5-\mathrm{mm}$ precision-drilled hole. Cross-sectional areas were measured at $1-\mathrm{mm}$ intervals and the volume of a segment was reconstructed by using a slice method. Slice
FIGURE 1. Lightwire assembly. The cone-shaped mirror is mounted in front of a $0.25-\mathrm{mm}$ graded-index fiber with the aid of a special bushing and transparent tubing:
FIGURE 2. Angioscopic view of a coronary cast with projected ring of light (left) and the manually traced outline (right) used for calculations.
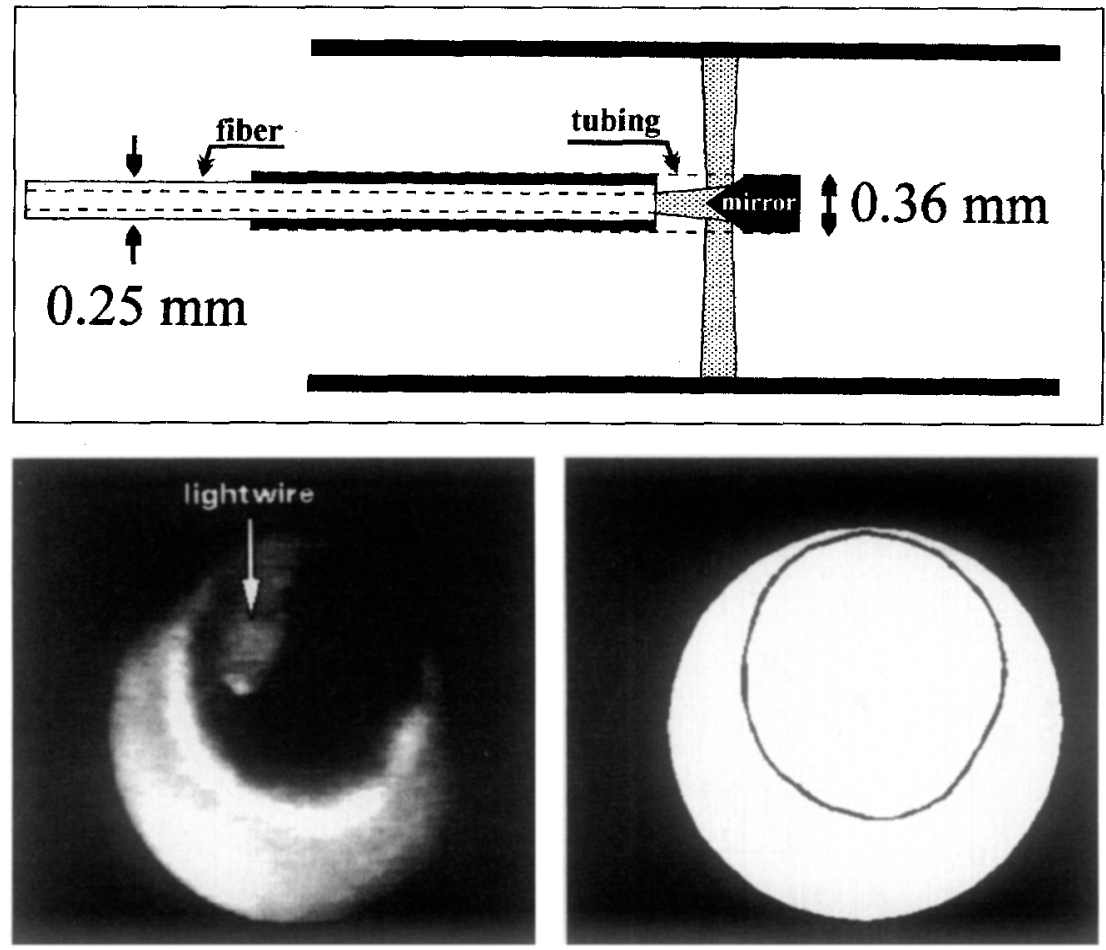


\begin{tabular}{|lcccccc|}
\hline \multicolumn{2}{|l|}{ TABLE I } & Analysis Success Score of Coronary Casts & & \\
\hline Cast & $\begin{array}{c}\text { Frames } \\
\text { Total }\end{array}$ & $\begin{array}{c}\text { Frames } \\
\text { Analyzed }\end{array}$ & $\begin{array}{c}\text { Segments } \\
\text { Total }\end{array}$ & $\begin{array}{c}\text { Segments } \\
\text { Analyzed }\end{array}$ & $\begin{array}{c}\text { Area, All Frames } \\
\mathrm{mm}^{2} \text { (mean } \pm \text { SD) }\end{array}$ & $\begin{array}{c}\text { Length, All Segments } \\
\text { mm (mean } \pm \text { SD) }\end{array}$ \\
\hline 1 & 67 & 41 & 7 & 2 & $8.49 \pm 0.77$ & $9.6 \pm 0.23$ \\
2 & 73 & 50 & 7 & 3 & $5.31 \pm 2.17$ & $8.1 \pm 0.63$ \\
3 & 65 & 65 & 14 & 14 & $8.27 \pm 1.13$ & $4.7 \pm 0.32$ \\
4 & 67 & 4 & 12 & 0 & $6.44 \pm 3.17$ & $5.6 \pm 0.19$ \\
Total & 272 & 160 & 40 & 19 & $7.13 \pm 1.52$ & $7.0 \pm 2.24$ \\
\hline
\end{tabular}

volume was defined as cross-sectional area $\times$ slice thickness $(1 \mathrm{~mm})$. At segment boundaries, the slice thickness was less than $1 \mathrm{~mm}$ and depended on the boundary position with respect to the 1-mm interval positions.

To evaluate the reproducibility of the area measurements, the series of 9 holes was analyzed 3 times by 2 observers. Volume reproducibility was evaluated by repeated $(n=3)$ acquisition and analysis of the cylindrical ceramic phantom.

Statistics: The data are expressed as mean signed difference \pm standard deviation. Linear regression analysis was applied to compare the segmental volume measurements with the true volumes.

\section{RESULTS}

The intraobserver variability in determining the crosssectional area (Figure 3) revealed a small scatter for both observers (standard error of estimate $[\mathrm{SEE}]=0.297$ and $0.293 \mathrm{~mm}^{2}$, respectively). The close agreement between the area measurements of both observers as expressed in the almost equal regression equations indicates also a small interobserver variability (Figure 3 ).

Repeated ( $\mathrm{n}=3)$ image acquisition and volume reconstruction on the cylindric phantom resulted in a mean deviation of the measured volume from true volume of $0.16 \pm 4.16 \%$. Linear regression analysis for those data showed good correlation (Figure 4, left). Volume reconstruction of the cone-shaped phantom resulted in a deviation of the measured volumes from the true volumes by $-1.6 \%$ and $1.4 \%$ for the cone-shaped segments and $11.5 \%$ for the cylindrical segment.

The true volumes of the 40 coronary segments measured with the microdispenser varied from 17 to $93 \mathrm{~mm}^{3}$ with total cast volume ranging from 297 to $570 \mathrm{~mm}^{3}$. From the video recordings of the 4 coronary casts, a total of 272 frames at $1-\mathrm{mm}$ intervals were selected for analysis. Because of incomplete visualization of the ring of light, only 160 frames were suitable for analysis (Table I). Subsequently, segmental volume reconstruction, which required the analysis of all frames within a segment, was possible in 19 of 40 segments. Linear regression analysis on the obtained volume data showed a good correlation between measured and true volumes $(r=0.97, y=$ $0.88 \mathrm{x}+6.58 \mathrm{~mm}^{3}, \mathrm{SEE}=3.48 \mathrm{~mm}^{3}$; Figure 4 , right .

\section{DIscussion}

For the evaluation of new therapeutic interventional techniques or characterization of endoluminal coronary morphology, conventional angiography has major limitations because of the characteristic shadow imaging technique. Several investigators have emphasized the potential advantage of angioscopic imaging of the coronary arteries over conventional angiography. ${ }^{1,2,4}$ However, a major limitation of angioscopy has been the absence of a quantification method. In principle, angioscopic images provide detailed 3-dimensional geometric and color information of the endoluminal surface. Quantitative assessment of this information would be quite useful. In this study, we tested the applicability of an earlier proposed lightwire ${ }^{7}$ in a more realistic way by investigating the feasibility of intraluminal volume reconstruction of casts from stenotic human coronary arteries. Under standardized conditions (e.g., after the administration of a vasodilator, measurement of intraluminal pressure, and adjustment of flush rate), volume reconstruction may be useful in evaluating short- or long-term results of coro-

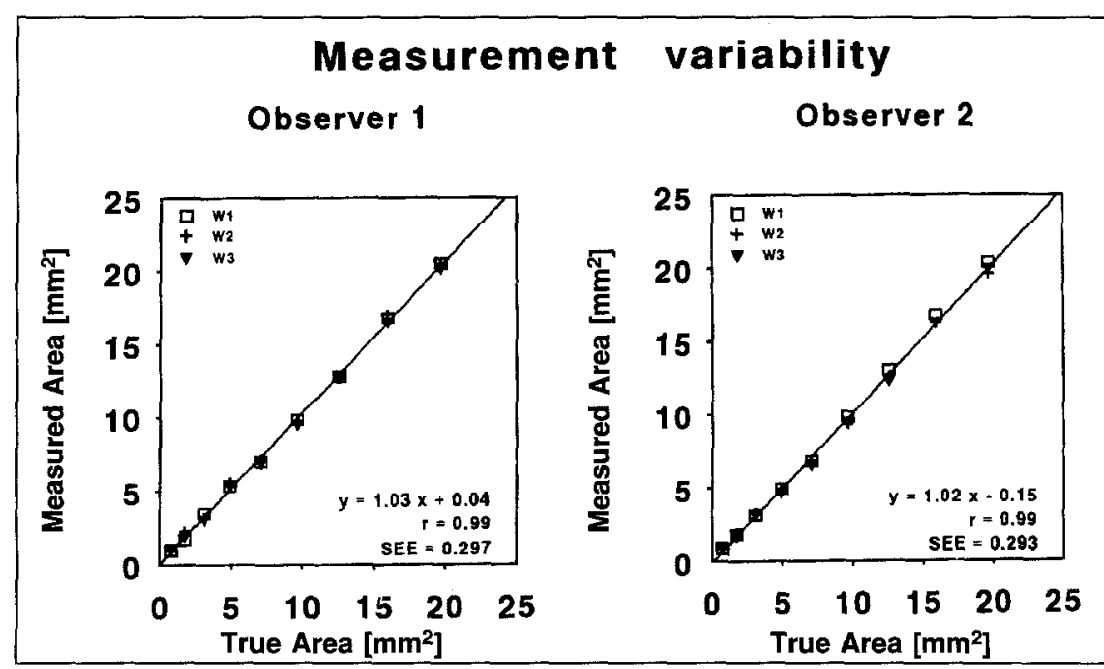

FIGURE 3. Area values of 9 precision-drilled holes measured by 2 observers are plotted against true area values. The measurements were repeated 3 times (W1, W2, and W3) by each observer. 
nary interventions. The small intra- and interobserver variabilities (Figure 3) demonstrate the high accuracy of this technique in estimating luminal cross-sectional area. Also, repeated determination of the segmental volumes of the cylindrical ceramic phantom itself showed a good correlation with the true segmental volumes and a small SEE (Figure 4).

Regression analysis on the coronary cast data showed a slightly increased SEE compared with the ceramic phantoms. A potential crror contributing to the increased scatter in the reconstruction of the coronary segmental volumes is variation in the actual speed of the angioscope advancing through the casts. This error accumulates due to the additionally required matching of the video recording with the actual segment positions, which are not identifiable in the images. Reconstruction errors may also occur when the implicit assumption that the angioscope and fiber are aligned parallel to the symmetry axis of the vessel does not hold true. The resulting error of such misalignment is related to the cosine of the intersection angle of catheter axis and vessel axis. The vessel segments selected for the preparation of the coronary casts were almost straight. For this reason, we do not expect a major contribution of this type of error to the current measurements. The relatively poor reflective properties of the thin white coating of paint may have contributed to the total error as well as to the low success rate in volume reconstruction. Under in vivo conditions, it might be expected that variations in the optical properties of plaque components affect the image of the ring of light due to scattered light from deeper tissue layers. This may require the use of shorter wavelengths that do not penetrate as deeply into the tissue. These subjects require additional study. Visual comparison of the ring of light between coated coronary casts and normal vessel wall showed a higher reflection of the vessel wall, suggesting an improvement of the visibility under in vivo conditions.

The most important reason for the relatively low success rate of the current set-up is the incomplete visualization of the ring of light when it is projected behind protruding structures (Figure 5, panels b, g, and h) or at relatively sharp local bends in front of the angioscope (Figure 5, panels $\mathrm{c}$ and d). To improve the visibility of the ring of light in vessels with severe luminal irregularity or near bends, the catheter tip to mirror distance should be minimized (Figure 5, panel h). The minimal lens-mirror distance depends on the viewing angle of the angioscope, the vessel diameter (Figure 5, panels e and $\mathrm{f}$ ), and also on the catheter position (Figure 5, panels $a$ and $b$ ). In our study, the mirror assembly was fixed to the angioscopic catheter, and the distance to the mirror was selected on the basis of the largest occurring diameter $(5 \mathrm{~mm})$ and assuming an eccentric catheter position. As a consequence, the low frequency of complete ring visualization in the casts does not reflect an optimal situation, particularly with respect to the smallest luminal diameters. Table I presents data on the low success rate in casts with severe luminal irregularity (indicated by a wide spread in mean luminal area [cast 4 , cast 2]) and in casts with very local stenotic sites combined with relatively long segment lengths (cast 1). Both these types of casts reduce the chance of successful reconstruction. Studies focusing on the measurement of, for example, minimal luminal cross-sectional area will show higher success rates because only a single area needs to be quantified. New lightwires with an adjustable, quantifiable lens-mirror distance should be designed for further investigations. If successful, this may greatly enhance the applicability of this quantification technique

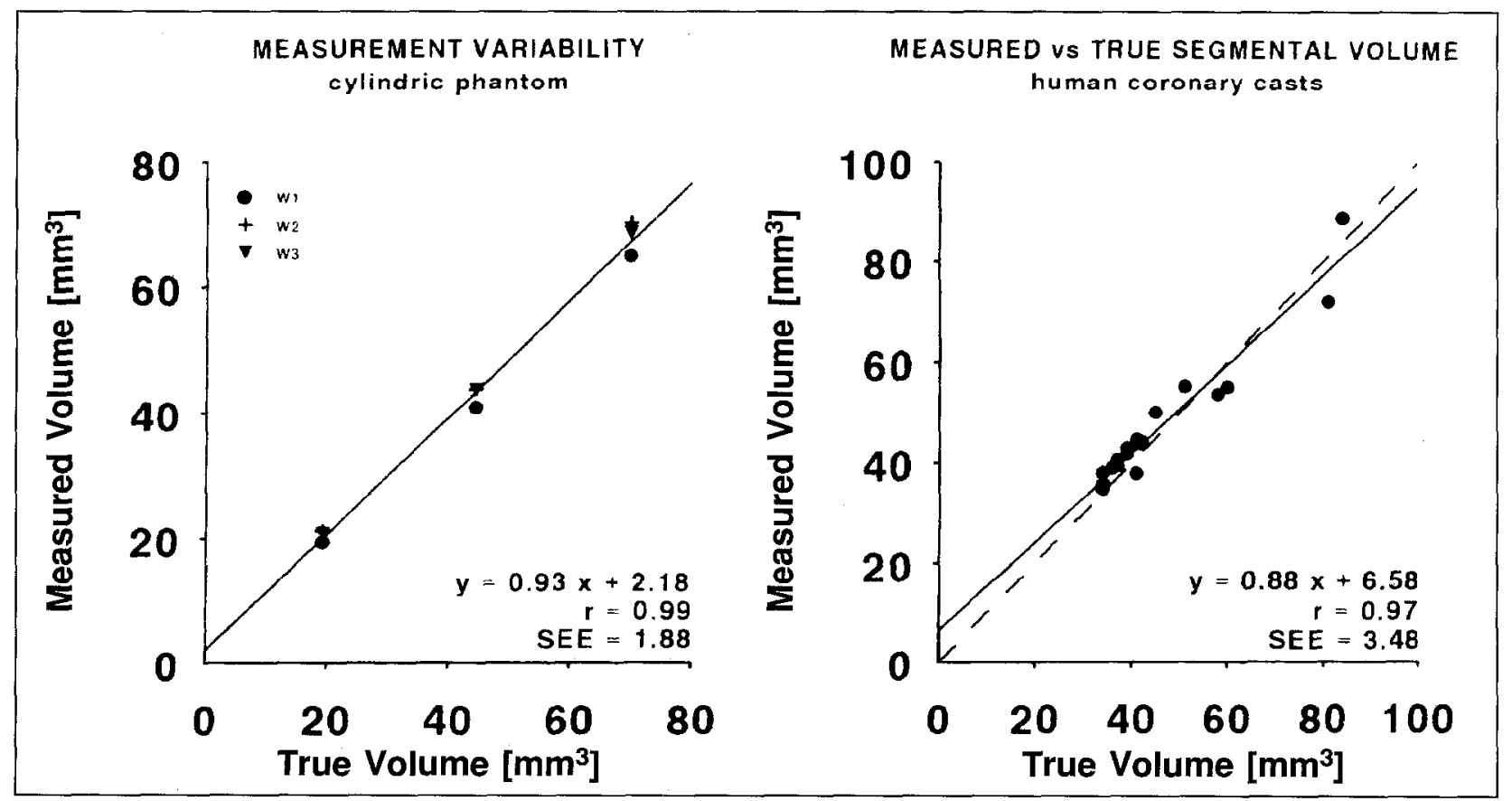

FIGURE 4. Measurement variability for repeated $(n=3)$ image acquisition and analysis (W1, W2, and W3) of the cylindrical ceramic phantom (left) and comparison between the angioscopically derived segmental volumes and the volumes measured with the microdispenser system (right). 


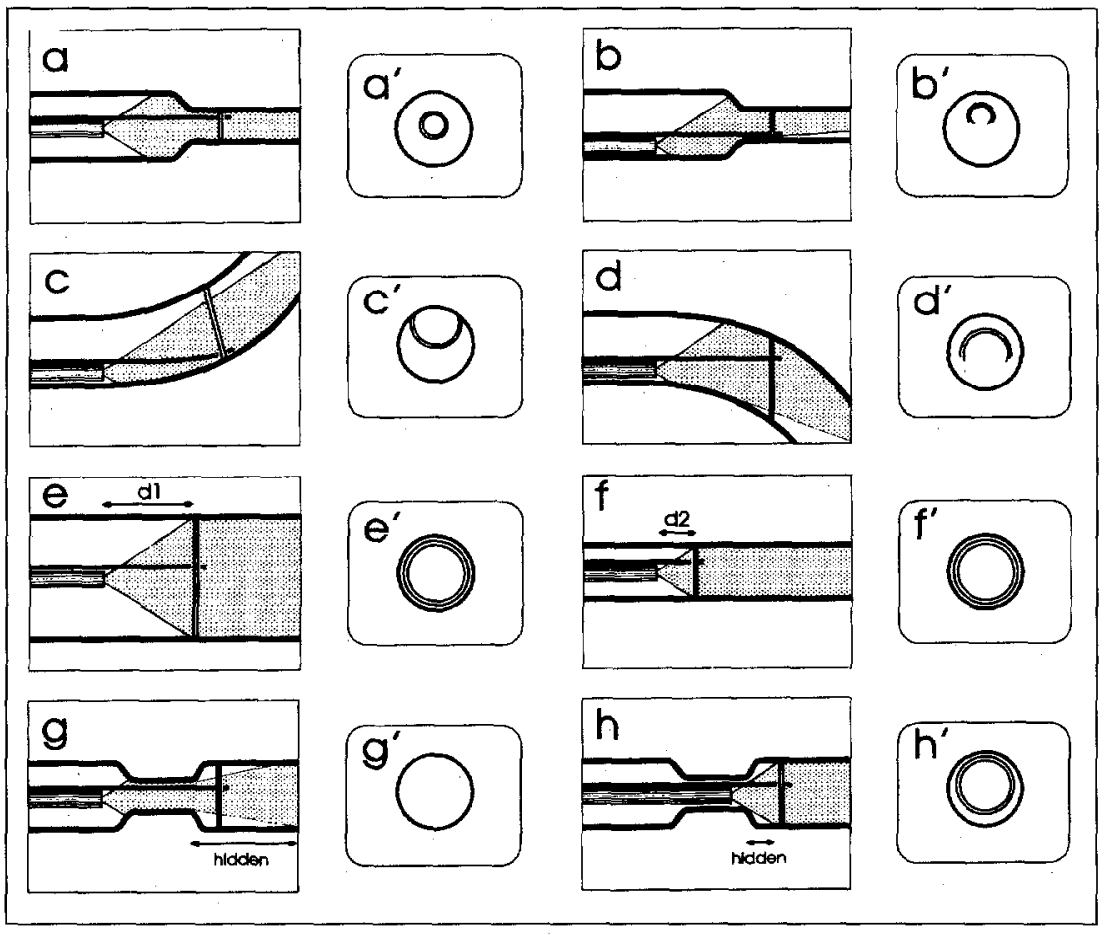

FIGURE 5. Positions for angioscope and lightwire. Panels $a^{\prime}$ to $h^{\prime}$ show the resulting ring of light in the angioscopic image. The dotted area is visualized by the angioscope. Complete visualization of the ring of light in a straight stenotic segment for a concentric catheter position is illustrated in panel a. Total or partial invisibility may occur behind stenoses (panel $\mathrm{g}$ ), near bends in front of the catheter (panels $c$ and $d$ ), or in the case of an eccentric catheter position (panel b). Optimal lens-mirror distance depends on field of view and vessel diameter for a central catheter position (panels $e$ and $f$ ). In vessels with protruding endoluminal structures, hidden space (panels g and $h$ ) will be minimized with a lens-mirror distance adapted to the actual luminal diameter (pane/ $h$ ).

in clinical practice. Besides volume reconstruction, other applications of this light-projection approach might become topics for research as well. For example, it might be possible to combine the geometric information with quantitative endoluminal surface color measurements at the site of the projected ring of light to provide a unique tool for 3-dimensional reconstruction of the endoluminal surface morphology in full color.

Acknowledgment: We thank Wim van Alphen for his assistance in developing the mirror assembly, Ir. Martin J. van der Hoek for his valuable advice on fiber optics, and Dr. Kenneth Lehmann for reviewing the manuscript.

1. Ramee SR, White CJ, Collins TJ, Mesa JE, Murgo JP. Percutaneous angioscopy during coronary angioplasty using a steerable microangioscope. J Am Coll Cardiol 1991;17:100-105.

2. Mizuno K, Miyamoto A, Satomura K, Kurita A, Arai T, Sakurada M, Yanagida $S$, Nakamura $H$. Angioscopic coronary macromorphology in patients with acute coronary disorders. Lancet 1991;337:809-812.

3. Sherman CT, Litvack F, Guundfest W, Lee M, Hickey A, Chaux A, Kass R, Blanche C, Matloff J, Morgenstern L, Ganz W, Swan HJC, Forrester J. Coronary angioscopy in patients with unstable angina pectoris. $N$ Engl J Med 1986;315: 913-919.

4. Uchida $Y$, Hasegawa $K$, Kawamura K, Shibuya I. Angioscopic observation of the coronary luminal changes induced by percutaneous transluminal coronary angioplasty. Am Heart J 1989;117:769-776.

5. Nakamura F, Kvasnicka J, Uchida Y, Geschwind HJ. Percutaneous angioscupic evaluation of luminal changes induced by excimer laser angioplasty. Am Heart I 1992;124:1467-1472.

6. White $\mathrm{CJ}$, Ramee $\mathrm{SR}$, Mesa $\mathrm{JE}_{\mathrm{r}}$ Collins $\mathrm{TJ}$. Percutaneous coronary angioscopy in patients with restenosis after coronary angioplasty. J Am Coll Cardiol 1991; 17:46B-49B

7. Spears RJ, Raza SJ, Ali M, Iyer GS, Cheong W, Crilly RJ. Quantitative angioscopy: a new method for measurement of luminal dimensions by use of a "lightwire" (abstr). J Am Coll Cardiol 1993;21:133A.

8. Doriot PA, Suilen C, Guggenheim N, Dorsaz PA, Chappuis F, Rutishauser W. Morphometry versus densitometry - a comparison by use of casts of human coronary arteries. Int J Card Imag 1992;8:121-130.

9. Haase J. Experimental validation and clinical comparison of quantitative coronary analysis systems. Doctoral The sis, Erasmus University, Rotterdam, 1993;189. 10. van der Borden SG, Oomen JAF, Slager CJ, Assmann P. Computer assisted echocardiographic analysis. A semi-automated approach. IEEE Comput Cardiol 1987:425-428. 